\title{
MOMOWo-LA CREATIVIDAD DE LAS MUJERES DESDE EL MOVIMIENTO MODERNO
}

\section{MoMoWo-Women's Creativity since The Modern Movement}

\author{
Esther Rodríguez Ortiz ${ }^{1}$ \\ Fecha de recepción: 30-03-2018 - Fecha de aceptación: 30-05-2018 \\ Hábitat y Sociedad (ISSN 2173-125X), n. ${ }^{\circ}$ 11, noviembre de 2018, pp. 249-256. \\ http://dx.doi.org/10.12795/HabitatySociedad.2018.i11.15
}

\begin{abstract}
The European project MoMoWo-Women's Creativity since the Modern Movement is financed by the European Union's Creative Europe Programme. It has as objective to make known the women works in professions related to designing. From an interdisciplinary perspective and Pan-European, MoMoWo considers that for the contemporaneity is relevant the work of women in Architecture, Industrial Design or Civil Engineering from a social and cultural point of view. The Project addresses the equal opportunities between men and women from the past until the actuality, and it aspires to stimulate and increment the value of the works and the gains achieved by women through the actual generations, as well as it aspires to strengthen the networks between women that work in the creativity field. Through the activities of the Project, not only it realizes a hard labor in that professional research areas, but also encourages the interaction between those professionals and the public in general.
\end{abstract}

\section{Key words}

MoMoWo; European project; Women architects; Civil engineers; Industrial design; Modern Movement

\section{Resumen}

El proyecto europeo MoMoWo-Women's Creativity since the Modern Movement, está patrocinado por el programa Europa Creativa de la Unión Europea y tiene como objetivo dar a conocer el trabajo de las mujeres en las profesiones relacionadas con el diseño. Desde una perspectiva interdisciplinar y paneuropea, MoMoWo considera relevante para la contemporaneidad, tanto desde un punto de vista social como cultural, el trabajo de las mujeres dentro de la Arquitectura, el Diseño Industrial e Ingeniería Civil. Desde el proyecto se aborda la igualdad de oportunidades tanto de hombres como de mujeres desde el pasado y la actualidad, además, aspira a estimular y a aumentar el valor de las obras como de los logros que consiguieron estas mujeres dándolas a conocer a las generaciones actuales, así como también aspira a fortalecer las redes entre las mujeres que trabajan en el ámbito de la creatividad. A través de las actividades propuestas por el proyecto, no sólo se realiza una ardua labor de investigación en esos ámbitos profesionales, sino que se promueve la interacción entre profesionales y el público en general.

Palabras clave

MoMoWo; Proyecto europeo; Arquitectas; Ingenieras civiles; Diseño industrial; Movimiento Moderno

\footnotetext{
1 Doctora en Historia del Arte por la Universidad de Oviedo (2015), ayudante de investigación en MoMoWo-Women's Creativity since
} the Modern Movement e investigadora del grupo de Arts and Crafts de la Universidad de Oviedo. E-mail: tetelaviana@hotmail.com 


\section{Introducción}

MoMoWo-Women's Creativity since the Modern Movement está formado por un consorcio de universidades y centros de investigación de seis estados miembros de la Unión Europea. Estas siete instituciones están especializadas en Estudios de Género, Patrimonio Cultural, Historia del Movimiento Moderno, Arquitectura, Restauración, Historia del Arte, Marketing, Turismo y Gestión Cultural. El resultado de la agrupación de las diferentes líneas de investigación es un grupo de trabajo que ofrece diferentes perspectivas en torno al objeto de estudio, que no es otro que el papel desempeñado por las mujeres en el ámbito de la arquitectura, el diseño industrial, el urbanismo, el paisajismo, el diseño de interiores y la ingeniería civil. Profesiones que las han estado reservadas y monopolizadas por los hombres y a las que las mujeres no han tenido acceso hasta bien entrado el siglo xx.

MoMoWo está liderado por la arquitecta Emilia Maria Garda, del Departamento de Ingeniería Estructural, Geotécnica y de Edificación - DISEG, del Politécnico de Turín, siendo los demás miembros del proyecto IADE-U, Institute of Art, Design and Enterprisa de Lisboa (Portugal); la Universidad de Oviedo, la Vrije University (Netherlands); el France Stele Institute of Art History, ZRC-SAZU (Eslovenia); Slovakia University of Technology, STUBA (Eslovaquia); el Istituto Superiore sui Sistemi Territoriali per l'innovazione de Turín. Formó parte de MoMoWo el Laboratoire de Recherche Historique en Rhône-Alpes de la Université Grenoble Alpes (Francia) hasta septiembre del 2017. El proyecto tiene una duración de 48 meses, tuvo su comienzo en octubre del 2017 y finalizará en octubre del 2018 y ha sido financiado con 1.157 .898 euros.

Uno de los aspectos más valorados del proyecto, no fue tanto la puesta en valor de las obras de estas mujeres profesionales, sino el puente que se traza entre las distintas generaciones de mujeres que se dedicaron a unas carreras profesionales destinadas a los hombres. Así, se vinculan pasado, presente y futuro a través de un proyecto cuya labor primordial es la de visibilizar y dar a conocer la actividad profesional de arquitectas, paisajistas, diseñadoras industriales, urbanistas e ingenieras civiles. Al destacar el valor que las obras de estas mujeres han aportado al mundo del diseño, MoMoWo espera empoderar y fortalecer a las futuras generaciones de mujeres creativas, que se mantienen activas en la actualidad y a las estudiantes que quieran desarrollar su carrera dentro de estos ámbitos profesionales.

MoMoWo, tal y como nos sugiere su título, toma como punto de partida el Movimiento Moderno en Europa, un momento de gran clamor cultural pero también un punto de quiebra político y social. Además, este punto de inflexión histórica representa el primer hito histórico en la liberación de las mujeres. Si tenemos en cuenta este último punto, debemos señalar que muchas de las mujeres que a principios del siglo xx querían desarrollar su labor profesional en países como Francia, Holanda, Alemania, Holanda, o Inglaterra, debían asociarse con sus maridos ya que por sí solas, aunque existieron casos, el camino no era tan fácil.

Entre los objetivos prioritarios del proyecto, en primer lugar, se encuentra el de reforzar la capacidad de los sectores culturales y creativos dirigiendo el foco a los grupos de mujeres que están poco representados dentro de los siguientes campos: Cultura, Arquitectura y Diseño (artes decorativas, interiores, industriales y diseños gráficos). En segundo lugar, promover la movilidad de mujeres arquitectas, diseñadoras, 
comisarias, historiadoras, estudiantes, restauradoras y profesionales del turismo, y estimular la circulación de sus obras en la Unión Europea con el objetivo de aumentar la conciencia, el conocimiento y el interés en el patrimonio cultural europeo creado por las mujeres.

Para lograr y conseguir esta labor de difusión y colaboración entre profesionales, el proyecto se configura a través de un ingente número de paquetes de trabajo que se concentran en la difusión de contenidos dirigida a diferentes audiencias, estimulando la participación de jóvenes diseñadoras, arquitectas etc., en diferentes disciplinas creativas. Otro de los cometidos es el de buscar una dimensión turística de los resultados, tratar de implicar a asociaciones profesionales y de grupos creativos, además de extender estas iniciativas al ámbito académico e investigador.

\section{Contenidos del Proyecto}

Uno de los primeros paquetes de trabajo desarrollados por MoMoWo fue el de sacar a concurso público la imagen corporativa de MoMoWo mediante un concurso internacional de diseño gráfico. Esta actividad, dirigida principalmente a jóvenes diseñadores, fue coordinada por ENSILIS-IADE de Portugal que en enero de 2015 sacó a concurso público la propuesta. Un jurado internacional mencionó al ganador públicamente durante la celebración del Festival dell'Architettura de Turín en 2015 y se aprovechó la ocasión para exponer todas y cada una de las propuestas recibidas impresas en tela y situadas como si de ropa limpia se tratara, buscando ese paralelismo simbólico que habla de un rol atribuido tradicionalmente a la mujer como es el de la lavandería, o las labores del hogar, como podemos apreciar en la Figura 1.

La ganadora fue la joven diseñadora Marisa Passos de Braga, Portugal. Una vez se tiene la imagen corporativa, se pone en marcha la página web a la que se incluyen redes sociales como Facebook, Twitter, Instagram y el blog del proyecto, que se mantienen especialmente activas y que se monitorizan periódicamente. ${ }^{2}$

Otro de los paquetes de trabajo fue la realización de itinerarios culturales-turísticos en varias localidades europeas, donde se ofrecían al público nuevas vías de interpretación del espacio urbano a través de las obras de arquitectas, ingenieras o diseñadoras. Se definieron 6 itinerarios en cada una de las siguientes ciudades: Lisboa, Barcelona, Turín, París, Holanda y Eslovenia. Los itinerarios, además, cuentan con códigos QR y AR ubicados delante de cada edificio para que el visitante consulte la información pertinente acerca de las autoras y sobre el proyecto desde cualquier dispositivo móvil mediante las aplicaciones al uso. Los libros impresos en papel se distribuyeron de manera gratuita en puntos de información local, oficinas de turismo y en las asociaciones profesionales de cada país.

Pero sin duda, uno de los paquetes de trabajo más laborioso es la realización de una extensa y completa base de datos de mujeres profesionales dentro del ámbito de la construcción y del diseño en Europa. Es esta una iniciativa compleja porque abarca todos los países europeos y no se circunscribe exclusivamente a los países representados en el consorcio. Esta base de datos cuenta con la inclusión de referencias bibliográficas, y es mucho más exhaustiva de las que podamos encontrarnos como usuarios de Internet. Debido a su compleja naturaleza y la propia extensión del mismo esta base de datos será presentada al final del proyecto en el que se realizará su difusión.

2 Cfr. www.momowo.eu 

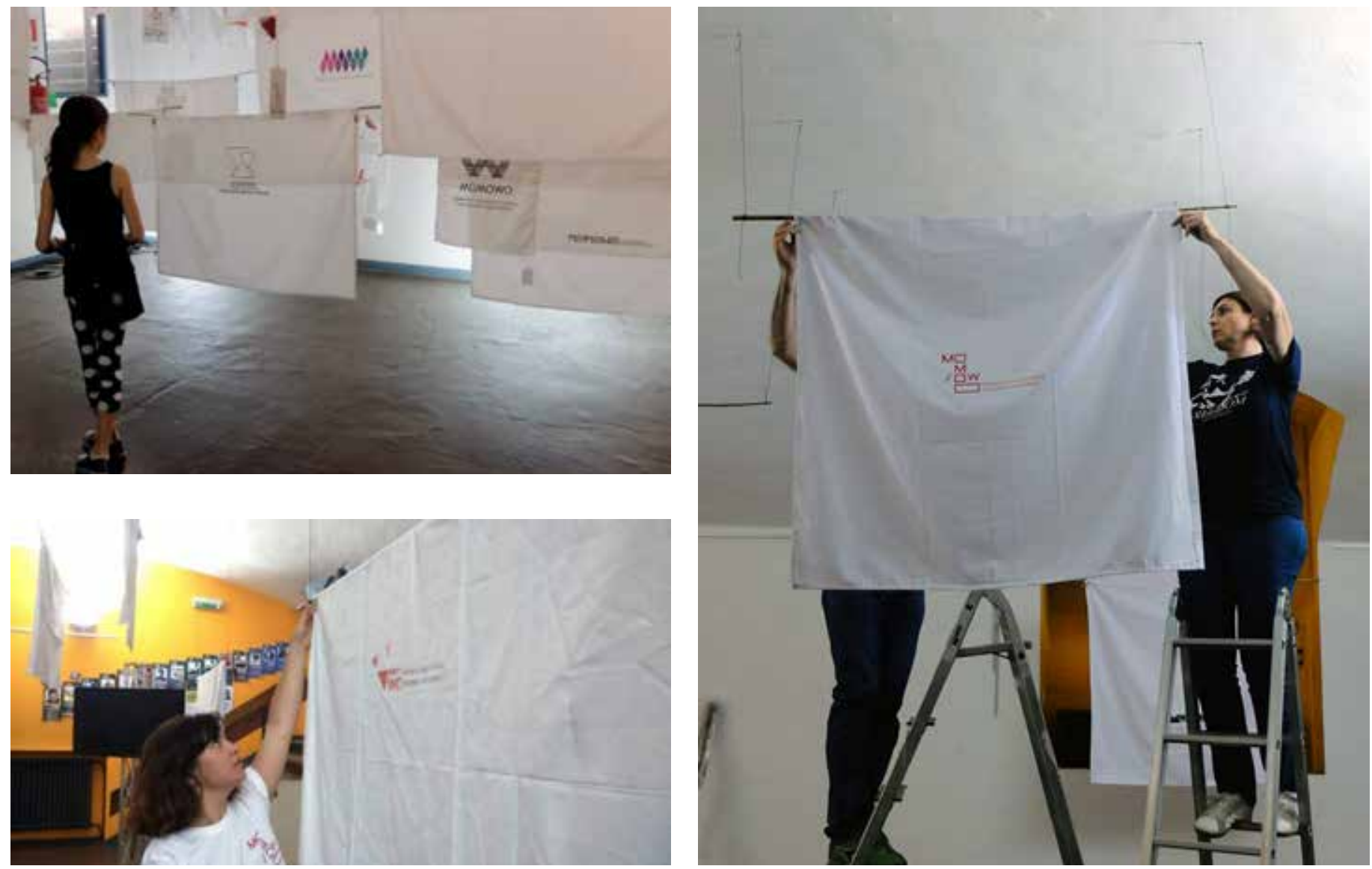

No debemos olvidarnos que los pilares de MoMoWo son académi-

Figura 1: Presentación de los diseños de concurso público para dotar de imagen corporativa al proyecto MoMoWo en el Festival dell'Architettura de Turín en julio 2015. Fuente: Elaboración Propia.
3 Cfr. "La Universidad acoge un congreso sobre creatividad y mujer" (2017, 03 de octubre). El Comercio. Recuperado el 11 de mayo de 2018 de: http:/ / www.elcomercio.es/culturas/universidad-acogecongreso-20171003002924-ntvo.html cos, y, por tanto, buena parte de sus paquetes de trabajo están basados en la investigación pura y dura. Con el fin de crear también una red de profesionales y especialistas académicos dentro del ámbito profesional que abarca el proyecto, se celebraron sendos congresos anuales. Así en septiembre de 2015 se celebró en Leiden (Holanda) el I International Conference-WorkShop, dirigido a trabajos relacionados con las mujeres en el diseño y la arquitectura desde 1918 hasta 1945. En octubre de 2016 tuvo lugar el segundo encuentro científico en el Research Centre of Slovene Academy of Sciences and Arts Slovenia, abarcando el periodo histórico desde 1946 hasta 1968. En octubre del 2017 tuvo lugar el III International Conference-Workshop organizado por la Universidad de Oviedo aceptando trabajos académicos sobre el rol de las mujeres desde 1969 hasta $1989 .{ }^{3}$ En junio del 2018, como colofón del proyecto, tendrá lugar en Turín, Italia, el Final Symposium de MoMoWo, cuyo reclamo virtual podemos ver en la Figura 2, que contará con la participación de arquitectas y profesionales de los diferentes sectores con carreras ya consolidadas y a estudiantes y expertos de todo el mundo.

Quizá desde el ángulo de la difusión, una de las actividades con mayor repercusión mediática fue la exposición internacional itinerante que, con el título "MoMoWo. 100 projects in 100 years. Women's architecture \& design in Europe (1918-2018)" (Figura 3), se inauguró en Oviedo (Asturias) en Julio de 2016 y ha recorrido cinco países europeos, además de encontrarse en vías de negociación con los Colegios Oficiales de Arquitectos de España para realizar un recorrido nacional en sus sedes, siendo los primeros en acogerla el Colegio Oficial de Arquitectos de Asturias y el Colegio Oficial de Arquitectos de Aragón. La exposición consta de tres partes: en una primera sección se analizan y visualizan una selección de obras de mujeres arquitectas y diseñadoras europeas desde 1918 hasta 


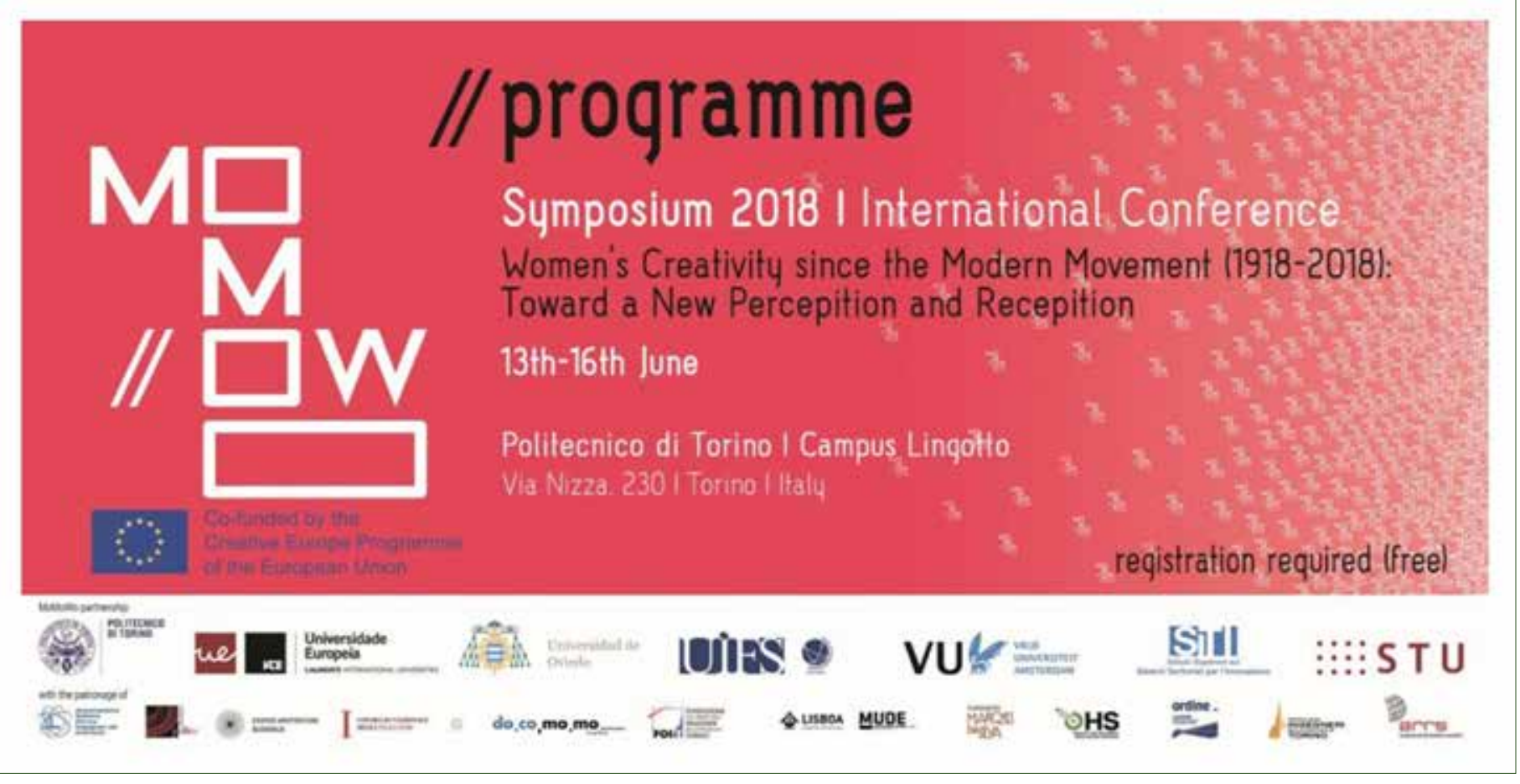

2017, desde una amplia panorámica de nacionalidades, del tipo de piezas y del carácter de los proyectos mismos. En una segunda sección aparece lo que se denominó como CronoMoMoWo, que no es otra cosa que un repertorio de cien años de conquistas sociales, políticas y culturales de las mujeres europeas en esos años y que se vincula con los logros de las actividades profesionales objeto del estudio, como la creación de las primeras asociaciones profesionales, los premios concursos de arquitectura y diseño, las primeras graduadas en escuelas de arquitectura, etc. En una tercera sección se exponen las fotografías ganadoras del concurso internacional de fotografía. La exposición quedó recogida en un catálogo que también ha sido repartido por las bibliotecas nacionales e internacionales de manera gratuita, acompañado de sendos artículos científicos escritos tanto por miembros del comité científico del proyecto como por jóvenes investigadores.
Figura 2. Banner Final Symposium de MoMoWo en Turín, junio 2018 . Fuente: Elaboración Propia.

Figura 3. Póster de la Exposición Internacional Itinerante. Fuente: Elaboración Propia.

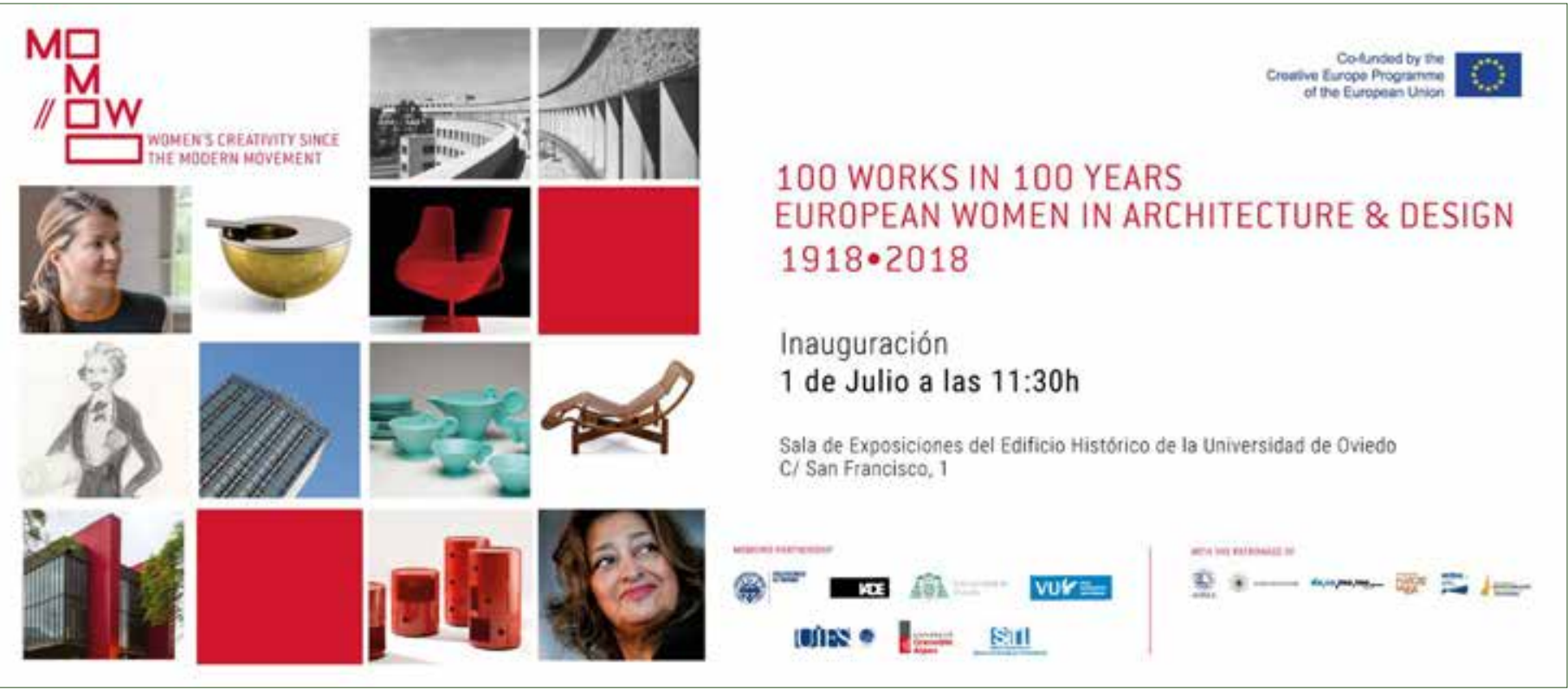




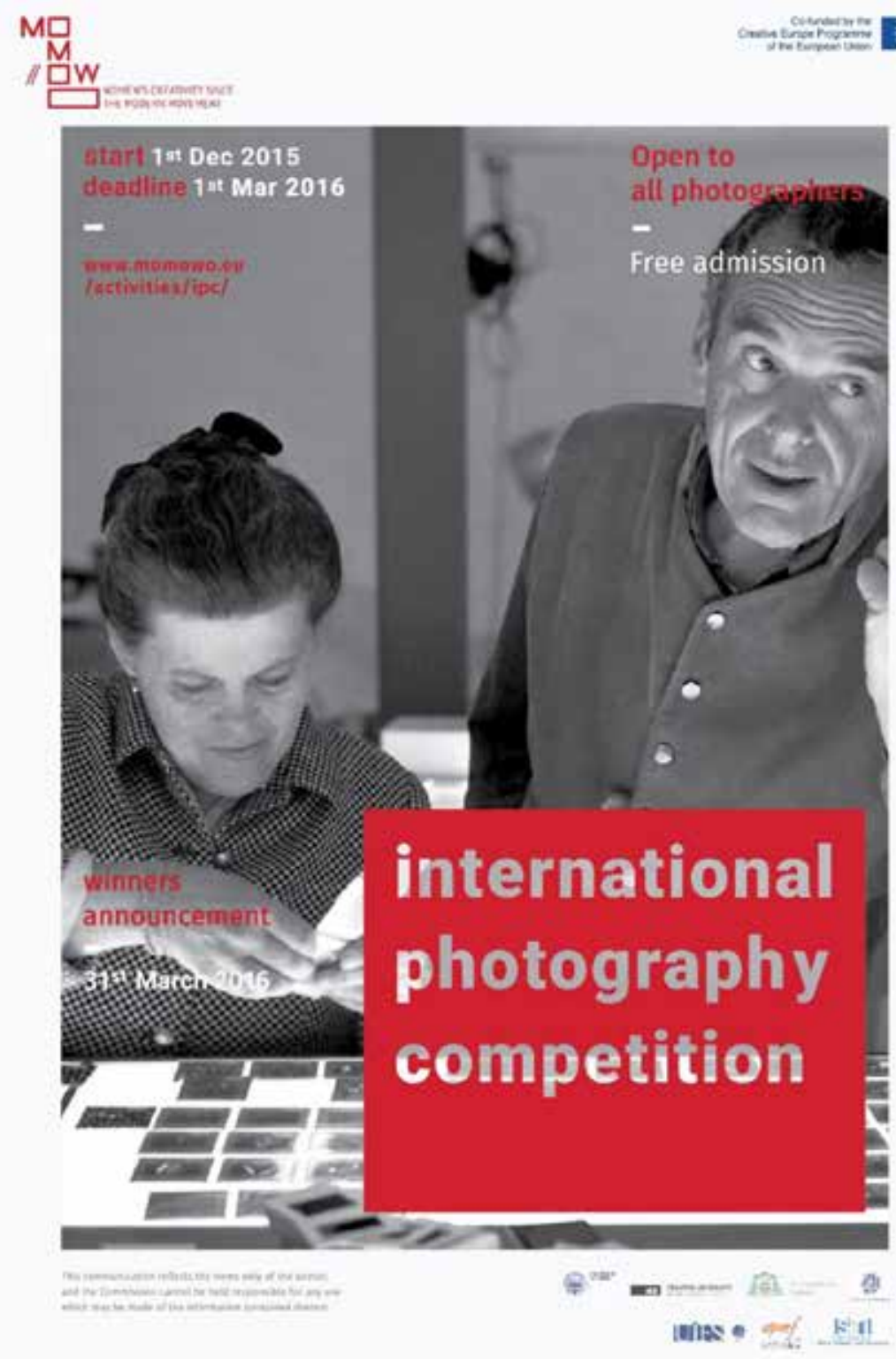

Otra de las actividades del proyecto que involucró a profesionales de otros sectores, en este caso el de la fotografía, fue la celebración del concurso internacional de fotografía (Figura 4). Este paquete de trabajo forma parte de la estrategia de difusión del proyecto. Se trataba de un concurso que pretendía promover el trabajo en equipo entre las profesionales del diseño y los profesionales de la imagen, pues son dos campos que se benefician recíprocamente. Una de las características esenciales es que no sólo estaba dirigido a los fotógrafos profesionales, sino que pudieron participar tanto estudiantes como aficionados. La misión del reportaje fotográfico era el de reflejar el carácter y el modo de vida de las diseñadoras y arquitectas dentro de su casa, siendo este espacio un lugar diseñado y creado por ellas. Esta iniciativa internacional fue una incomparable plataforma de proyección profesional tanto para los fotógrafos como para las profesionales, ya que los trabajos ganadores fueron incluidos en el catálogo de la exposición itinerante, y se convirtieron en objetos que pasaron a formar parte de la exposición.

Con motivo del Día Internacional de los Derechos de la Mujer, el 8 de marzo, MoMoWo propuso como actividad el Open Day o Jornadas de Puertas Abiertas en los estudios de arquitectas, diseñadoras e ingenieras civiles. Esta iniciativa se

Figura 4: Póster de la Competición Internacional de Fotografía, $2015-2016$ Fuente: Elaboración Propia. llevó a cabo simultáneamente en Lisboa, España, París, Eslovenia y Turín, y se convirtió en una actividad pionera en cuanto a la actividad en sí misma se refiere y a la simultaneidad en su desarrollo en diferentes países de la Unión Europea. Se trata de una jornada en la que todas aquellas profesionales del sector que están interesadas en participar en esta actividad abren las puertas de su estudio al público en general no sólo para explicar en qué consiste su labor profesional, sino también para contar qué dificultades se han encontrado a la hora de desarrollar su trabajo como mujeres en una profesión tan masculinizada como la que tratamos. Lo que se pretende con esta iniciativa, y se consigue, es dar visibilidad a las profesionales dentro de su propio territorio, pues muchas de ellas aún viven a la sombra profesional. Es también una oportunidad para animar a las jóvenes estudiantes a introducirse dentro de los campos de la arquitectura, el diseño o la ingeniería para formarse como futuras profesionales. Añadir además que el primer año la participación de las arquitectas fue algo tímida, en el último año se consiguieron abrir más de 100 estudios en los países miembros del proyecto, calando esta actividad entre las profesionales y el público que ha mostrado y muestra su interés por las distintas propuestas. 


\section{A modo de conclusión}

MoMoWo a través de sus actividades y su apuesta por la perspectiva de género no sólo ha sabido visualizar el rol de las mujeres dentro de la arquitectura, el diseño o la ingeniería civil, rescatando del olvido aquellas profesionales que trabajaron codo a codo con sus maridos, pero que fueron absorbidas por un apellido, sino que además ha servido como espejo para las nuevas generaciones de mujeres que intentan abrirse paso en un, cada vez menos, ambiente profesional masculinizado. Pero además y por supuesto, ha servido como lanzadera a través de la cual se han abierto numerosas nuevas vías de investigación que, sin duda, abrirán otras posibilidades de futuro académico. 


\section{Referencias}

Blanco, L. (2017). Nuevos cimientos frente al patriarcado. La Nueva España, 30 de octubre de 2017. Recuperado el 13 de junio de 2018, de: http:/ / www. lne.es/oviedo/2017/10/03/nuevos-cimientos-frente-patriarcado/2172325.html.

Fernández García, A., Franchini, C., Garda, E. et al. (eds.) (2016). MoMoWo. 100 Works in 100 Years European Women in Architecture and Design. 1918-2018. Ljubljana: Stele Institute of Art History ZRC SAZU. Recuperado el 11 de mayo de 2018, de: http:/ / www.momowo.eu/wp-content/uploads/2015/01/ MoMoWo_Catalog_final.pdf.

Groot, M., Seražin, H., Franchini, C. et al. (eds.)
(2017). MOMOWO: Women Designers, Craftswomen, Architects and Engineers between 1918 and 1945. Ljubljana: Založba ZRC. Recuperado el 11 de mayo de 2018, de: https:/ / omp.zrc-sazu.si/zalozba-zrc/cata$\log /$ book $/ 2$.

Levi Sacerdotti, S., Seražin, H., Garda E. et al. (eds.). (2016). MoMoWo. Women Architecture Ẽ Design Itineraries across Europe. Ljubljana: Stele Institute of Art History ZRC SAZU. Recuperado el $11 \mathrm{de}$ mayo de 2018, de: https:/ / www.dropbox.com/s/ sjc34wgq6481ar6/GUIDEBOOK_MOMOWO_high. pdf?dl=0.

Rodríguez Ortiz, E. (2018). MoMoWo-La creatividad de las mujeres desde el Movimiento Moderno. Hábitat y Sociedad, 11, 249256.

<http://dx.doi.org/10.12795/HabitatySociedad.2018.i11.15> 